\section{Case Reports in Neurology}

Case Rep Neurol 2020;12:24-26

DOl: 10.1159/000505229

Published online: January 15, 2020

(C) 2020 The Author(s)

Published by S. Karger AG, Base

www.karger.com/crn

This article is licensed under the Creative Commons Attribution-NonCommercial 4.0 International License (CC BY-NC) (http://www.karger.com/Services/OpenAccessLicense). Usage and distribution for commercial purposes requires written permission.

\title{
Stiff Person Syndrome and Acetylcholine Receptor Ganglionic Neuronal Antibodies
}

\author{
Jacobo Lester ${ }^{a} \quad$ Jimmy Cojab $^{b} \quad$ Enrique Klériga ${ }^{c}$ \\ aDepartment of Neurology, Instituto Mexicano de Neurociencias, Huixquilucan, Mexico; \\ bInternal Medicine Service, Hospital Angeles Lomas, Huixquilucan, Mexico; \\ 'Department of Neurosurgery, Instituto Mexicano de Neurociencias, Huixquilucan, Mexico
}

\section{Keywords}

Stiff person · Ganglionic neuronal antibodies · Antibodies

\begin{abstract}
The association between stiff person syndrome and paraneoplastic syndromes has been described, linking intracellular or extracellular antibodies. We describe the case of a 64-year-old woman with stiff person syndrome and positivity for acetylcholine receptor ganglionic neuronal antibodies, which can also be seen in muscular hyperexcitability conditions, as well as other paraneoplastic syndromes. The relevance of this report is the possible direct elevation of this antibody due to a direct immunological cause. Acetylcholine receptor ganglionic neuronal antibodies have been reported with autoimmune autonomic neuropathy and paraneoplastic syndromes linked to lung cancer and myastheniform syndromes, as well as in some muscular hyperexcitability states, but not in stiff person syndrome.

(C) 2020 The Author(s) Published by S. Karger AG, Basel
\end{abstract}

\section{Introduction}

Acetylcholine receptor ganglionic neuronal (AChRGN) antibodies (Ab) have been reported with autoimmune autonomic neuropathy $[1,2]$ and paraneoplastic syndromes linked to lung cancer and myastheniform syndromes [1], but also in some muscular hyperexcitability states [1]. 


\section{Case Report}

A 64-year-old woman, without a relevant family history, reported having had rigidity while walking 4 years before her consultation, involuntary tongue movements, biting of the oral mucosa, as well as a "jumping" sensation of her abdomen.

She was evaluated by a movement disorder specialist. A diagnosis of tardive dyskinesia was done, and she was started on tetrabenazine $25 \mathrm{mg}$ t.i.d. without any improvement, quitting after 1 year of treatment.

Two and a half years before, her walking became more difficult, the abdomen was stiff, it jumped and arched, she reported hardness in the muscles of the neck, face and trunk, and a walking pattern "like a robot." She consulted another specialist in movement disorders, who requested multiple studies: the glutamic acid decarboxylase 65 (GAD65) Ab were $2.5(<0.02)$, the rest of the $\mathrm{Ab}$ being negative.

She consulted with us for the first time in 2017, complaining of jerky movements, with stiff sensation of her neck and trunk with hyperextension; moreover, she mentioned a stiff abdomen, postural tremor, trunk and limb action, as well as "robotic gait."

We requested an electromyography that revealed the presence of intermittent contractions of the phasic type at rest, at a frequency of $6 \mathrm{~Hz}$ in virtually all the evaluated muscles. No co-contraction of agonists and antagonists was detected. There was a slight decrease in motor unit recruitment and reflex hyperexcitability of spinal motoneurons, especially in lower limbs and brainstem (blink).

Based on the complete clinical presentation and complementary studies, the diagnosis of stiff person syndrome (SPS) was considered. She was started on diazepam $10 \mathrm{mg}$ t.i.d. and baclofen $20 \mathrm{mg}$ t.i.d., without improvement in the following 2 weeks; after that we initiated clonazepam $2 \mathrm{mg}$ b.i.d., also without any clinical improvement. Knowing that rigidity had not improved within a 2-year time lapse, we decided to give her intravenous immunoglobulin 0.4 $\mathrm{g} / \mathrm{kg}$ for 5 days, a 5 -day treatment with plasmapheresis and 2 sessions of rituximab and cyclophosphamide, $500 \mathrm{mg} /$ each in a 4-day frame, with partial improvement, mostly on her gait.

A full-body positron emission tomography was performed in 2019, which showed a small right mammary zone which was biopsied, resulting in a benign lesion.

New immunological and paraneoplastic laboratories requested concluded positivity for AChRGN Ab $0.06(<0.02)$ and GAD65 Ab $9(<5)$, with the rest being negative, emphasizing antineuronal nuclear Ab (ANNA)-1, ANNA-2, ANNA-3, antiglial nuclear Ab (AGNA)-1, Purkinje cell Ab (PCA)-1, PCA-2, PCA-Tr, amphiphysin Ab, CRMP-5-IgG, striational muscle Ab, P/Q type calcium channel, $\mathrm{N}$-type calcium channel, Ach receptor $\mathrm{Ab}$, and neuronal $\mathrm{K}+$ channel $\mathrm{Ab}$.

\section{Discussion}

SPS may be associated with immunological or paraneoplastic syndromes [1-3]. The presence of tumoral and immunologic $\mathrm{Ab}$ has been described in SPS. These Ab may be intra- or extracellular [3].

Until now, we have ruled out paraneoplastic causes in our patient. We have centered our focus just on the immunological ones, mentioning N-methyl-D-aspartate receptor in encephalitis with early psychiatric and cognitive features, leucine-rich glioma-inactivated 1 with brief dystonic movements, contactin-associated protein-like 2 that are juxtaparanodal protein $\mathrm{Ab}$, with multiple neuromyotonic choreic, ataxic, and myoclonic movements, aquaporin-4 and myelin oligodendrocyte glycoprotein with the optic neuromyelitis spectrum, and IgLON5- 
associated sleep behavior disorder with bulbar involvement. GAD65 Ab were mostly detected in SPS and clinically associated with a fluctuating muscle stiffness, muscle spasms, and hyperreflexia [3].

Our patient's AChRGN Ab was positive, usually detected in about $50 \%$ of patients with autoimmune autonomic neuropathy [1,2], but it can also be seen in about $5-10 \%$ of patients with lung cancer, Lambert-Eaton myastheniform syndrome, myasthenia gravis, and thymoma [1].

It is considered that AChRGN Ab could be physiologically detected in muscular hyperexcitability like in some neuromyotonias, cramp-fasciculations and acquired rippling muscle syndrome [1].

As far as we are aware of, AChRGN Ab have not been reported in SPS. We hypothesize that these antibodies might be elevated due to a direct immunological cause. However, it could also occur because of a hyperexcitability state caused by severe stiffness due to the patient's own condition.

More studies should be done in patients with similar characteristics to investigate our hypothesis and eventually determine if it should be used as an additional marker in SPS.

\section{Statement of Ethics}

This article is a case report; only patient consent was obtained, but it was not presented to an ethics committee. The patient granted verbal and written consent.

\section{Disclosure Statement}

The authors declare that there are no additional disclosures to report.

\section{Funding Sources}

No specific funding was received for this work.

\section{Author Contributions}

J.L.: conception, organization, execution, writing the first draft, review and critique.

J.C.: organization, design, execution, review and critique.

E.K.: conception, design, review and critique.

\section{References}

1 Vernino S, Lennon VA. Neuronal ganglionic acetylcholine receptor autoimmunity. Ann N Y Acad Sci. 2003 Sep;998(1):211-4.

2 Watanabe E, Fujita T, Shimono M, Koike H, Yasumoto S, Hirose S. Recurrent autonomic and sensory neuropathy in a patient with anti-ganglionic acetylcholine receptor antibodies. eNeurologicalSci. 2018 Aug;12:36-8.

3 Damato V, Balint B, Kienzler AK, Irani SR. The clinical features, underlying immunology, and treatment of autoantibody-mediated movement disorders. Mov Disord. 2018 Sep;33(9):1376-89. 